\section{FMH für Psychiatrie und Psychotherapie: Mehr Klarheit tut not - Hier ist sie!}

\section{Y. Maurer}

Was gilt heute noch, 2 Jahre nach der Inkraftsetzung des Weiterbildungsprogramms für den Erwerb des Facharzttitels FMH für Psychiatrie und Psychotherapie?

Wie war das gemeint mit der Stundenanzahl? Wer muss wozu sein Einverständnis geben oder wo haben Assistenzärztinnen und Assistenzärzte freie Wahl?

Wie verhält es sich mit den genannten «drei anerkannten psychotherapeutischen Modellen»? - Eine graphische Darstellung soll Klarheit schaffen.

Um mehr Klarheit in diese Fragen zu bringen, die sich vorwiegend auf den Paragraphen 2.3 des «Weiterbildungsprogramms FMH Psychiatrie und Psychotherapie" beziehen (d.h. 2.3.1-2.3.7), habe ich mich bemüht, die Anforderungen graphisch zu gestalten (Tab. 1). Diese kann auch für weitere Diskussionen und kleinere oder grössere Revisionen dienlich sein.

Das vorliegende Weiterbildungsprogramm (WBP) kann im Grunde nur von denjenigen im wahren Sinn und Zweck erfasst werden, die die Entstehungsgeschichte kennen, die die Vorentwürfe zur Kenntnis genommen und die Diskussionen an den Versammlungen der SGP mitverfolgt haben. Das WBP ist ein Kompromisspapier, ein Versuch, viel Wünschenswertes unter einen Hut zu bringen und neue mögliche Entwicklungen in Ausbildungs- und Therapieansätzen nicht gleichzeitig im Keime zu ersticken.

Viele Jahrzehnte lang war die Schweiz eine wichtige Metropole psychiatrischer und vor allem psychotherapeutischer Ansätze. Speziell die Gegend um Zürich hat sich mit damals "avantgardistischen" Ansätzen nicht nur in der Schweiz, sondern auch im Ausland Weltruhm geholt. So kann die Schweiz auf eine lange und bedeutende Tradition psychotherapeutischer Ausbildung für Ärztinnen und Ärzte, Psychiaterinnen und Psychiater und nichtärztliche Psychotherapeuten verweisen.

Korrespondenz:

Dr. med. Yvonne Maurer

Institut für Körperzentrierte Psychotherapie

Kanzleistrasse 17

CH-8004 Zürich
Gemäss meinen Erkundigungen ist diese Tradition privater Ausbildungsinstitute für die Suisse Romande weniger charakteristisch. Dies bedeutet also, dass bereits die Ausgangslage in verschiedenen Kantonen für eine Regelung (gemäss $2.3 \mathrm{im}$ WBP) unterschiedlich ist. Von diesem Standpunkt aus wäre eine dezentrale Lösung nötig. Dies hat allerdings den Nachteil, dass der Austausch zwischen den verschiedenen psychiatrischen Institutionen und nicht zuletzt der Stellenwechsel der Assistenzärztinnen und Assistenzärzte dadurch drastisch erschwert wird. Zudem wirkt es auf einen Patienten seltsam, wenn sie oder er in einem Kanton oder in einer Klinik ganz anders behandelt wird als z.B. früher in einer andern.

Dies hat inzwischen vielen eingeleuchtet, namentlich ärztlichen Direktorinnen und Direktoren von psychiatrischen Kliniken und Polikliniken in der Schweiz. Der Wunsch vieler geht dahin, eine möglichst breite Therapiepalette anbieten zu können.

In krassem Widerspruch dazu steht nun die Einengung des "psychotherapeutischen Angebots im engeren Sinn" auf die im WBP aufgeführten "drei Modelle».

Anlässlich der Diskussionen um diese zu enge Einteilung wurden viele Gegenstimmen laut, z.B. auch von Prof. R. Battegay, Basel.

$\overline{\text { Es geht um seriöse Psychotherapie-Weiterbildungs- }}$ institute

Um zu verstehen, weshalb es auf diesem Hintergrund dennoch zu einer beschränkten Einteilung von Psychotherapieformen auf bloss drei Modelle kam, muss man um die Problematik wissen, dass sich die SKWF (bzw. die SGP) nicht auf eine aufwendige Zertifizierung von Weiterbildungsinstituten einlassen wollte. Die Absicht der SKWF war und ist, eine breite Palette von Weiterbildungsangeboten zuzulassen und dennoch die Möglichkeit offenzuhalten, besonders ungeeignete Ausbildungsinstitute bzw. -institutionen auszuschliessen. $\mathrm{Zu}$ den "ungeeigneten" wären z.B. nicht seriöse, nicht (von bedeutenden Gremien) anerkannte, nicht bereits langjährig bewährte Ausbildungsinstitute mit grösserem akademischem Dozentinnen- und Dozententeam zu rechnen. Es war angesichts der ausserordentlichen Vielfalt von Psychotherapierichtungen der ausdrückliche Wunsch, eine Regelung zu finden, die möglichst offen formuliert war, und nicht eine abschliessende Positiv- bzw. Negativliste zu erstellen.

\section{Zerrbildinformation der drei Modelle}

Obiges wurde offenbar zu wenig kommuniziert, sodass wir heute eine Zerrbildinformation und grosse Ratlosigkeit bei den Assistenzärztinnen und Assistenzärzten feststellen müssen sowie auch bei einigen Verantwortlichen von psychiatrischen Institutionen, die den Entscheidungsfindungsprozess der letzten Jahre in der SGP nicht selbst mitgemacht haben. Über das weitgefasste Verständnis der "drei» Modelle muss 


\section{Tabelle 1}

Weiterbildungsprogramm FMH für Psychiatrie und Psychotherapie.

Punkt 2.3 des WBP als Tabelle, erstellt von Dr. med. Y. Maurer, IKP, Zürich, und durchgesehen vom Präsidenten der ständigen Kommission für Weiter- und Fortbildung SKWF, Dr. med. Hp. Wengle.

\begin{tabular}{|c|c|c|c|c|}
\hline WBP & Ort & Stunden & Organisiert durch & $\begin{array}{l}\text { Einverständnis oder } \\
\text { vorgeschrieben durch }\end{array}$ \\
\hline 2.3.1 & $\begin{array}{l}\text { Theoretisch-praktischer } \\
\text { Unterricht, regionales Angebot; } \\
\text { 50\% Psychiatrie, } \\
\text { 50\% Psychotherapie }\end{array}$ & 330 & $\begin{array}{l}\text { Regionaler Verbund der } \\
\text { Weiterbildungssstätten (Zürich, Basel, } \\
\text { Bern, Lausanne, Genf) }\end{array}$ & $\begin{array}{l}\text { Leiterin/Leiter der } \\
\text { Weiterbildungsstätte }\end{array}$ \\
\hline 2.3.1 & $\begin{array}{l}\text { Theoretisch-praktischer } \\
\text { Unterricht, freie Wahl; } \\
\text { 50\% Psychiatrie, } \\
50 \% \text { Psychotherapie }\end{array}$ & $310^{1}$ & $\begin{array}{l}\text { - Psychiatrie: diverse, } \\
\text { - Psychotherapie: z. B. private Psycho- } \\
\text { therapieinstitute (gem. einem Modell) }\end{array}$ & $\begin{array}{l}\text { Freie Wahl der/des } \\
\text { Assistenzärztin/-arztes, } \\
\text { Validierung durch Leiterin/Leiter } \\
\text { Weiterbildungsstätte }\end{array}$ \\
\hline 2.3 .5 & $\begin{array}{l}\text { weiteres Modell, } \\
\text { zusätzlich weiteres Modell } \\
\text { (siehe Text unten) }\end{array}$ & $\begin{array}{l}12 \\
12\end{array}$ & $\begin{array}{l}\text { Regionaler Verbund der } \\
\text { Weiterbildungsstätten (Zürich, Basel, } \\
\text { Bern, Lausanne, Genf oder andere) }\end{array}$ & $\begin{array}{l}\text { Freie Wahl der } \\
\text { Assistenzärztinnen/-ärzte, } \\
\text { Validierung durch Leiterin/Leiter } \\
\text { der Weiterbildungsstätte }\end{array}$ \\
\hline 2.3.6.1 & $\begin{array}{l}\text { Psychiatrische oder } \\
\text { psychotherapeutische Supervision } \\
\text { nach freier Wahl }\end{array}$ & $50^{2}$ & $\begin{array}{l}\text { Leiterin/Leiter der Weiterbildungsstätte } \\
\text { oder private Psychotherapieinstitute }\end{array}$ & Assistenzärztin/-arzt \\
\hline 2.3.6.2 & Psychiatrische Supervision & 125 & Leiterin/Leiter der Weiterbildungsstätte & $\begin{array}{l}\text { Leiterin/Leiter der } \\
\text { Weiterbildungsstätte }\end{array}$ \\
\hline 2.3.6.3 & $\begin{array}{l}\text { Psychotherapeutische } \\
\text { Supervision: } 3 \text {-10 Fälle, } \\
\text { Einzel- oder Gruppensupervision }\end{array}$ & $125^{3}$ & $\begin{array}{l}\text { Private Psychotherapieinstitute, } \\
\text { Psychotherapeutinnen/-therapeuten }\end{array}$ & $\begin{array}{l}\text { Leiterin/Leiter der } \\
\text { Weiterbildungsstätte in Absprache } \\
\text { mit Assistenzärztin/-arzt }\end{array}$ \\
\hline & Total Stunden & $964^{4}$ & & \\
\hline $\begin{array}{l}\mathrm{F} \\
\mathrm{e}\end{array}$ & \multicolumn{4}{|c|}{$\begin{array}{l}\text { Für die theoretisch-praktische Weiterbildung in Psychotherapie i.e.S. stehen also } 155 \text { Stunden zur Verfügung, vermittelt z.B. durch } \\
\text { ein privates Psychotherapieinstitut. }\end{array}$} \\
\hline 2,3 & \multicolumn{4}{|c|}{$\begin{array}{l}\text { Für die psychotherapeutische Supervision stehen } 125 \text { Stunden bzw. max. } 175 \text { Stunden zur Verfügung, vermittelt z.B. durch ein } \\
\text { privates Psychotherapieinstitut. }\end{array}$} \\
\hline r & \multicolumn{4}{|c|}{$\begin{array}{l}\text { Von den } 964 \text { Stunden sind mind. } 509 \text { Stunden für Assistenzärztinnen und -ärzte frei wählbar (310 Stunden 2.3.1, } 24 \text { Stunden 2.3.5, } \\
50 \text { Stunden 2.3.6.1 und } 125 \text { Stunden 2.3.6.3). } \\
\text { Von diesen 509 Stunden können unter obengenannten Bedingungen insgesamt mind. } 354 \text { Stunden an einem privaten Psychothe- } \\
\text { rapieinstitut absolviert werden (179 Stunden theoretisch-praktischer Unterricht [2.3.1 und 2.3.5] und } 175 \text { Stunden Supervision }\end{array}$} \\
\hline
\end{tabular}

besser informiert werden und dies wird befreiend wirken. Die «3» Modelle sind als Quadratur des Kreises zu betrachten und fokussieren mehr auf die Güte und wissenschaftliche Ausrichtung einer psychotherapeutischen Weiterbildung als deren theoretische "Modelletikette».

Zu einer Zeit, in der psychotherapeutische Modelle viel mehr als früher konvergieren, wäre es auch unsinnig, Zulassung und Abschluss von seriösen Psychotherapie-Weiterbildungsinstituten auf eine Diskussion der Modellzuteilung ihres Angebotes zu verschieben. Damit würde man auch Weiterentwicklungen in Richtung integrativ-ganzheitlicher Modelle behindern, was unserer Schweizer Tradition mit dem bio-psychosozialen Modell (auch als ganzheitliches Psychotherapie-Modell verstanden!) sehr widerspräche.

Das Weiterbildungsprogramm wollte und sollte auch an die Eigeninitiative der Assistenzärztinnen und -ärzte appellieren, nach Möglichkeiten der Weiterbildung zu suchen, die ihnen hinsichtlich Menschsein und gefühlsmässig entsprechen und sie Behandlungserfolge erleben lassen.
So sind in 2.3.1 «310 Stunden Unterricht im Rahmen des Lernzielkatalogs" - der "weiss Gott» fast zu viel enthält - "nach freier Wahl zu absolvieren». Viele Assistenzärztinnen und -ärzte - vielleicht sogar einige Chefärztinnen und Chefärzte - scheinen diese Formulierung überlesen zu haben. Und viele realisieren nicht, dass die obgenannte Quadratur des Kreises bezüglich der drei Modelle auch viele weitere angesehene und von der SKWF als geeignet erachtete Weiterbildungsinstitute für ihre Weiterbildung beinhaltet.

\section{Psychisches, körperliches und beziehungsmässiges Erleben integrieren}

Als Gesamtleiterin des Instituts für Körperzentrierte Psychotherapie (IKP) (Zürich, Bern, Basel) kenne ich die beschriebene Situation am IKP eben am besten und nenne daher dieses Beispiel: Im Ansatz gehört die von mir zusammen mit meinem Dozentinnen- und Dozententeam vor rund 20 Jahren begründete und lau- 
fend weiterentwickelte Körperzentrierte Psychotherapie IKP zur Strömung humanistischer Psychotherapie, in die auch Gestalttherapie, viele systemische und körperorientierte Psychotherapieansätze eingeordnet werden. Die Körperzentrierte Psychotherapie IKP ist eine Ganzheits-Psychotherapie, die von Theorie und Methode her systemisch ist und körperliches und beziehungsmässiges Verhalten erlebnisorientiert in Diagnose und Therapie einbezieht. Aus meiner mehr historischen Perspektive könnte man auch von einer ins Körperliche und Systemische hinein weiterentwickelten Gestalttherapie sprechen. Wir haben unseren Psychotherapieansatz wiederholt mittels IKPTagungen und Kongressen öffentlich und zusammen mit vielen Dozentinnen und Dozenten psychiatrischer Kliniken zur Diskussion gestellt [1-4]. Müsste unser Ansatz in die "Zwangsjacke» der drei Modelle gesteckt werden, wäre er am ehesten dem systemischen Modell zuzuordnen.

Das Spezielle an unserem Ansatz ist das Verbinden des therapeutischen Gesprächs mit aktiver und passiver Körperarbeit im Sinne des verbalen/ nonverbalen «Übersetzens» und Rückübersetzens [4]. Unser Vorgehen bietet die Möglichkeit, psychisches, körperliches und beziehungsmässiges (soziales) Erleben $\mathrm{zu}$ integrieren und das Bewusstgewordene auch im Körpergedächtnis zu verankern. Mehrere Publikationen sowie quantitative und qualitative wissenschaftliche Studien unterstreichen die hohe therapeutische Wirksamkeit unseres ganzheitlichen Ansatzes.

In unserem Beispiel sind wir seit vielen Jahren sowohl - was die wenigsten Assistenzärztinnen und -ärzte wissen dürften - von der Schweizer Charta für Psychotherapie, aber auch von diversen europäischen Gremien.

Das Dozententeam am IKP (zurzeit rund 40 gut ausgebildete Personen) rekrutiert sich mittlerweile aus der ganzen Schweiz, z.T. aus dem Ausland. Wir legen Wert auf Kostentransparenz der Ausbildung. Unsere vorgeschlagene Weiterbildung (die gemäss SKWF die Bedingungen für eine FMH-Anerkennung erfüllt) ist vergleichsweise günstig (zurzeit rund Fr. 3900.- pro Jahr (bei einer 3jährigen berufsbegleitenden Ausbildungszeit). Dies ist auch darauf zurückzuführen, dass wir auf bereits bestehende Infrastrukturen, langjährige Ausbildungserfahrung und anerkannte Ausbildnerinnen und Ausbilder zurückgreifen können.

Über Internet www.ikp-therapien.com kann mehr über die Weiterbildung zum Facharzt FMH für Psychiatrie und Psychotherapie eingesehen werden. Zusätzlich bieten wir - dank unserer Grösse und der weiteren Ausbildungszweige - ein breites Seminarangebot an, das allen Interessenten offensteht.

\section{Werden nur körperablehnende Ärztinnen und Ärzte Psychiaterinnen und Psychiater?}

Interessanterweise bildete das IKP bisher mehr Psychologinnen und Psychologen als Ärztinnen und Ärzte zu Psychotherapeutinnen und -therapeuten weiter. Hat dies spezielle Gründe? «Nur körperablehnende Ärzte werden Psychiater», sagte mir kürzlich eine Medizinerin, die ihr Fremdjahr in einer psychiatrischen Klinik macht. «Die althergebrachte Identität der Psychiaterinnen und Psychiater ist nur zu sprechen und nicht körperlich zu berühren, wie dies beispielsweise Chirurgen, Gynäkologen, Urologen selbstverständlich tun. Daher haben angehende Psychiaterinnen und Psychiater vielleicht weniger Interesse an einer ganzheitlichen Psychotherapieform, die zusätzlich den Körper in den therapeutischen Prozess einbezieht. Dafür sind doch die Physiotherapeutinnen und -therapeuten zuständig!» Ja, so dachte und handelte ich selber auch, als ich 1971 meine Dissertation über "Massage bei Depressiven - eine klinische Studie" in Angriff nahm: Ich "sprach" und sie (die "Physiotherapeutinnen und -therapeuten») be-«hand»-elten. Längst ist dies bei mir ganz anders geworden: Ich spreche, wir sprechen, spüren in den Körper, versuchen, das unerwünschte Gefühl mit dem Körper darzustellen und das gewünschte körperlich neu zu erleben und im Körpergedächtnis zu verankern. Bei allen zu längerer Dauer neigenden Störungen beziehen wir die Lebenspartnerinnen und -partner (oder die Familie) mit ein und bleiben auch da nicht nur auf der "Kopfebene», sondern lassen das Paar zusätzlich gegenseitig nonverbal kommunizieren, um besser zu erkennen, was zwischen ihnen läuft und neues Verhalten einzuüben [3, 5-8].

Wer sich als Ärztin bzw. Arzt verantwortlich fühlt, nach einem modernen und ganzheitlichen Stil zu diagnostizieren und zu therapieren, für die oder den wäre ein Psychotherapieansatz wie derjenige des IKP bestens geeignet.

\section{Literatur}

1 Maurer Y. Körperzentrierte Psychotherapie. Ganzheitlich orientierte Behandlungskonzepte und Therapiebeispiele. Stuttgart: Hippokrates (neu Zürich: IKP): 1993.

2 Maurer Y (Hrsg.). Bedeutende Psychotherapieformen der Gegenwart. Stuttgart: Hippokrates (neu Zürich: IKP); 1985.

3 Maurer Y. Der ganzheitliche Ansatz in der Psychotherapie. Wien, New York: Springer; 1999.

4 Maurer Y (Hrsg.). Grundlegende Theorien und Aspekte. Körperzentrierte Psychotherapie IKP. Zürich: IKP Verlag; 1998.

5 Maurer Y. Körperzentrierte Psychotherapie IKP: GanzheitsPsychotherapie bei Angstzuständen. Ther Umsch 1997; 54:585-90.

6 Maurer Y. Körperzentrierte Psychotherapie IKP: GanzheitsPsychotherapie. In: Pöldinger W (Hrsg.). Ganzheitliche Psychiatrie. Ther Umsch 1996;53:217-24.

7 Maurer Y. Angst hat viele Erscheinungsformen und bedarf ganzheitlicher Therapie: Denken wir immer daran? Schweiz Ärztezeitung 1990;71:1217-8.

8 Maurer Y. Was ist Körperzentrierte Psychotherapie IKP? Schweiz Ärztezeitung, 1985;66:1527-9. 